\title{
ANÁLISE DE PROPOSTA ALTERNATIVA NA OBTENÇÃO DO FATOR DE VISÃO DE CÉU POR MÉTODO FOTOGRÁFICO
}

\author{
MIRANDA, Soneize Auxiliadora de - soneize@gmail.com \\ Universidade do Estado do Mato Grosso / UNEMAT
}

PAULA, Diana Carolina Jesus de - arqdiana.paula@gmail.com

Universidade Federal do Mato Grosso / UFMT

MUSIS, Carlo Ralph - carlo.demusis@gmail.com

Universidade Federal do Mato Grosso / UFMT

NOGUEIRA, José de Souza - nogueira@ufmt.br

Universidade Federal do Mato Grosso / UFMT

\section{SANTOS, Flávia Maria de Moura - flavia_mms@hotmail.com Universidade Federal do Mato Grosso / UFMT}

NOGUEIRA, Marta Cristina de Jesus Albuquerque - mcjanp@gmail.com Universidade Federal do Mato Grosso / UFMT

\begin{abstract}
RESUMO: O fator de visão do céu (FVC) é apontado como uma das variáveis explicativas nas alterações microclimáticas do ambiente urbano. Neste contexto, o presente estudo objetivou analisar o desempenho de diferentes equipamentos para estimativa do FVC em diferentes alturas, obtidos com lentes olho-de-peixe de baixo custo, acopladas a câmera fotográfica de smartphone, e os valores de referência obtidos com camera fotográfica e lente olho-de-peixe profissionais. O método empregado foi composto por: levantamento em campo das imagens hemisféricas; estimativas do FVC por meio do programa RayMan, avaliação quantitativa e qualitativa do FVC, obtidos com o conjunto alternativo em relação ao equipamento de referência. De acordo com os resultados alcançados, a alteração do fator altura do ponto de visão não reportou diferenças significativas entre os valores do FVC, entretanto a variação do fator ângulo de visão das propostas alternativas reportaram diferenças significativas em comparação aos valores do FVC obtidos com o equipamento de referência.
\end{abstract}

PALAVRAS-CHAVE: Fator de visão do céu, Radiação Solar, Obstrução do céu.

\section{ANALYSIS OF ALTERNATIVE PROPOSAL IN OBTAINING THE SKY VISION FACTOR BY PHOTOGRAPHIC METHOD}

ABSTRACT: The sky vision factor (SVF) is indicated as one of the explanatory variables in the microclimatic alterations of the urban environment. In this context, the present study aimed to analyze the performance of different equipment for estimating SVF at different heights, obtained with low-cost fisheye lenses coupled to a smartphone photo camera, and the reference values obtained with a photographic camera and professional fisheye lens. The method used was: field survey of hemispheric images; SVF estimates using the RayMan program, quantitative and qualitative evaluation of SFV, obtained with the alternative set in relation to the reference equipment. According to the results obtained, the alteration of the factor of height of the point of view did not report significant differences between the SFV, however the variation of the angle of vision factor of the alternative proposals reported significant differences in comparison to the SFV values obtained with the equipment of reference.

KEYWORDS: Sky view factor, solar radiation, sky obstruction 

DEL CIELO POR MÉTODO FOTOGRÁFICO

RESUMEN: El factor de visión del cielo (FVC) es señalado como una de las variables explicativas en las alteraciones microclimáticas del ambiente urbano. En este contexto, el presente estudio objetivó analizar el desempeño de diferentes equipos para estimación del FVC en diferentes alturas, obtenidos con lentes ojo de pez de bajo costo, acopladas a la cámara fotográfica de smartphone, y los valores de referencia obtenidos con cámara fotográfica y lente de ojo de pez profesional. El método empleado fue compuesto por: levantamiento en campo de las imágenes hemisféricas; estimaciones del FVC a través del programa RayMan, evaluación cuantitativa y cualitativa del FVC, obtenidos con el conjunto alternativo en relación al equipo de referencia. De acuerdo con los resultados alcanzados, la alteración del factor altura del punto de visión no reportó diferencias significativas entre los valores del FVC, sin embargo la variación del factor ángulo de visión de las propuestas alternativas reportaron diferencias significativas en comparación a los valores del FVC obtenidos con el equipo De referencia.

PALABRAS CLAVE: Factor de visualizacion del cielo, radiacion solar, obstruccion del cielo

\section{ANALYSE DE PROPOSITION ALTERNATIVE POUR OBTENIR LE FACTEUR DE VISION DU CIEL PAR DE MÉTHODE PHOTOGRAPHIQUE}

RÉSUMÉ: Le facteur du ciel (CVF) vue est considérée comme l'une des variables explicatives dans les changements micro-climatiques dans l'environnement urbain. Dans ce contexte, cette étude visait à analyser les performances des différents dispositifs pour estimer la CVF à des moments différents, obtenu avec un objectif fish-eye à faible coût, couplé téléphone appareil photographique, et les valeurs de référence obtenues avec la caméra et objectif fisheye professionnel. La méthode utilisée était la suivante: étude sur le terrain des images hémisphériques; CVF estime par programme Rayman, l'évaluation quantitative et qualitative de CVF, obtenue avec l'autre ensemble par rapport à l'équipement de référence. Selon les résultats obtenus, le changement de point de vue du facteur de hauteur n'a signalé aucune différence significative entre les valeurs de CVF, mais la variation du facteur d'angle de vision des alternatives proposées ont rapporté des différences significatives par rapport aux valeurs CVF obtenues avec l'équipement de référence.

MOTS CLÉ: Facteur De Visualisation Du Ciel, radiation solaire, obstruction du ciel

\section{INTRODUÇÃO}

A conformação morfológica do tecido urbano, associada às propriedades de materiais construtivos e às atividades antropogênicas, tem impacto significativo no equilíbrio do balanço energético do ambiente urbanizado (OKE, 1981). Fatores como a orientação geográfica, densidade volumétrica, rugosidade e proporção geométrica dos espaços intra-urbanos, determinam o grau de interação com as variáveis atmosféricas, gerando condições microclimáticas diversificadas (NAKATA-OSAKI, et al. 2016).

Pesquisas remetem a importância da configuração dos cânions urbanos, expressa pela relação entre altura e largura $(H / W)$ e o percentual de céu visível, definido como o fator de visão de céu (FVC), sendo apontado como uma das variáveis explicativas nas alterações microclimáticas do meio urbano. $O$ valor do FVC é adimensional e varia entre 0 a 1, correspondendo a total obstrução do céu até a máxima parcela de céu visível sem qualquer obstáculo, respectivamente (OKE, 1981; CHAPMAN, 2007; SOUZA, et al. 2003; MATZARAKIS E MATUSCHEK, 2011). 
As relações entre o FVC e o comportamento da temperatura e umidade do ar têm por referência as pesquisas desenvolvidas por OKE (1981), que observou a influência da geometria urbana e o papel do FVC na formação de ilhas de calor urbana (ICU) noturna em condições atmosféricas estáveis. Estudos posteriores consolidaram a relevância da relação entre valores do FVC e a variação na intensidade máxima das ICU em diferentes configurações e escalas do clima urbano (SOUZA et al. 2010). Contudo, o grau da relação entre ICU e FVC depende de diferentes fatores, como orientação dos cânions urbanos, a ventilação e a presença de vegetação, associados às características do clima local, conforme observado em Alcoforado et al. (2009) e Nakata-Osaki, et al. (2016).

A estimativa do FVC pode ser obtida por métodos gráficos com base em representação geométrica da máscara de obstrução de um determinado ponto de observação; por método fotográfico com levantamento em campo para obtenção de imagens hemisféricas, utilizando-se lente grande angular de $180^{\circ}$ (olho-de-peixe), ou ainda, por meio de dados de um Sistema de Informações Geográficas (SIG), associados a programas computacionais específicos para o processamento das imagens e estimativa do FVC (CHAPMAN, 2007; SOUZA, 2003).

Atualmente, o método fotográfico e o uso de um SIG são recorrentes na definição do FVC em áreas urbanas, com aplicação conforme a especificidade de cada objeto de pesquisa. Vilela et al. (2009) analisaram a evolução da morfologia em área de expansão urbana por meio de levantamento em campo e simulação tridimensional, utilizando o método fotográfico para obtenção do FVC da área consolidada e um SIG para obtenção do FVC proporcionado pela futura expansão da malha urbana. Ribeiro et al. (2010) realizaram a representação tridimensional de uma complexa área urbana consolidada, onde a estimativa do FVC foi obtida por meio de SIG, que possibilitou uma análise detalhada da relação entre o FVC e o nível de sombreamento nos espaços intra-urbanos.

Para estudos do conforto térmico em espaços abertos, Chapman (2007) argumenta que o levantamento em campo por método fotográfico é mais adequado, por possibilitar avaliação mais precisa da obstrução do céu, incluindo a copa das árvores quando presentes em cânions urbanos. Neste sentido, Grimmond et al. (2001), Matzarakis et al. (2011) e Amorim et al. (2014) destacaram como aspectos positivos do método fotográfico, a possibilidade do registro de todos os elementos do entorno, incluindo a vegetação com maior precisão e agilidade, quando comparado ao método gráfico ou com SIG, que necessitam de maior número de dados, infraestrutura mais específica de hardware, software e maior capacidade de processamento de dados.

A definição por um determinado método está relacionada ao objeto e aos objetivos da pesquisa, às limitações impostas pela morfologia da área de estudo e a definição dos procedimentos metodológicos. Outro fator que pode influir na escolha do método adequado à pesquisa é a disponibilidade e acesso aos equipamentos apropriados. No método fotográfico um aspecto relatado com relativa frequência é a dificuldade enfrentada por pesquisadores no acesso ao equipamento apropriado, devido ao reduzido número de equipamentos, em consequência do elevado custo financeiro, que limita a disponibilidade desses equipamentos a um maior número de pesquisadores, (AMORIM, 2014; MINELLA, 2009). 
Neste sentido, algumas alternativas foram analisadas por Grimmond et al. (2001), que avaliaram o FVC de cânions urbanos obtido por diferentes câmeras profissionais, analógica e digital com lentes olho-de-peixe, e os valores obtidos por meio do analisador de dossel vegetal (LAI-2000), que estima a fração de céu disponível à entrada da radiação difusa. Entre os equipamentos os resultados apresentaram alto grau de correção, R2 de 0,8 a 1,0. Embora, os valores obtidos por meio do LAI-2000 tenham apresentado tendência de superestimar os valores do FVC para as áreas avaliadas.

L. Minella et al. (2009a) apresentaram uma técnica para compensar o fator de corte da imagem hemisférica obtida com câmera fotográfica digital stand, sem o sensor Full Frame. Os resultados foram satisfatórios com erro médio de $-0,31 \%$ em comparação aos valores do FVC obtidos a partir das imagens de uma câmera digital profissional.

Amorim et al. (2014) avaliaram alternativas com foco na redução do custo financeiro e na ampliação do emprego do FVC em estudos urbanos. Utilizando três alternativas para obter o FVC em um ponto da área de estudo, a simulação em 3D com os programas Sketchup Pro e Autodesk Ecotect, e a imagem hemisférica obtida por meio de uma lente olho-de-peixe de baixo custo, acoplada a um smartphone. Na análise dos valores do FVC em cada método simulado, nos programas 3D e no fotográfico com lente alternativa, apresentaram valores muito próximos, com diferença máxima de $2 \%$.

Os resultados apresentados reforçam a relevância em ampliar a investigação para as alternativas de baixo custo. Observa-se que a qualidade da imagem hemisférica é resultante do conjunto óptico, câmera fotográfica e lente, ou seja, ajustes do fator de corte ou enquadramento podem amenizar a falta do sensor Full Frame em uma câmera não profissional. O bom desempenho da lente olho-de-peixe de baixo custo observado pelos autores justifica ampliar a abrangência da investigação, com maior número de observações em diferentes configurações do espaço urbano.

Neste contexto, a presente pesquisa objetivou analisar o desempenho de diferentes equipamentos para estimativa dos valores do FVC, obtidos com lentes olho-de-peixe de baixo custo, acopladas a câmera fotográfica de smartphone e os valores de referência obtidos com câmera fotográfica e lente olho-de-peixe profissionais.

\section{ESTIMATIVA DO FVC POR MÉTODO FOTOGRÁFICO}

O tratamento das imagens hemisféricas digitalizadas é a primeira etapa do método fotográfico, que por meio de programas de edição de imagens podem ser realizadas as correções de contraste, brilho, saturação de cores ou a conversão em monocromático. Assim, tornando a área de céu visível em branco e a área obstruída em preto. Quanto maior a qualidade da imagem fotográfica maior será a precisão na definição da área obstruída pelos planos edificados, equipamentos urbanos e a vegetação (GRIMMOND et al., 2001).

No método fotográfico alguns fatores podem ter influência no resultado final do FVC, como a altura do ponto de visão do céu, ou da captura da imagem em relação ao solo. Por definição geométrica quando o ponto está acima do solo o plano horizontal do observador é deslocado, o que pode reduzir a altura 
efetiva dos elementos de obstrução em relação à área visível de céu (CHAPMAN, 2007; SOUZA et al. 2003).

Grimmond et al. (2001) analisaram o FVC obtidos por método fotográfico a 1,40m do solo, em relação aos valores do FVC estimados por meio de uma equação, considerando o ponto de visão no solo. Observaram que o efeito proporcionado pela variação na altura do ponto de visão foi mais significativo em cânions urbanos com menor altura $(H)$ em relação à largura (W). Svensson (2004) analisou a variação da temperatura do ar noturna em relação os valores do FVC obtidos a 2,0m de altura em 16 estações fixas e ao nível do solo em pontos de medições por transecto móvel, em diferentes configurações de cânions urbanos. O fator altura apresentou relação com a variação dos valores do FVC, no nível de significância de 5\%.

Outro fator que pode influenciar na estimativa do FVC é o ângulo de abertura das lentes olho-de-peixe. Um ângulo de abertura de $180^{\circ}$ possibilita o registro da área a partir do nível do solo, isto é, a imagem obtida de um edifício seria da base sobre a calçada até o topo da cobertura. Ocorre que, para algumas lentes o ângulo real pode variar para mais ou para menos. Desta maneira, Grimmond et al. (2001) testaram diferentes lentes de $180^{\circ}$, cujos valores reais foram de $148^{\circ}, 160^{\circ}, 189^{\circ}$ e $180^{\circ}$. Em avaliação comparativa com o ângulo real de $180^{\circ}$, para os valores do FVC o erro médio absoluto foi de $0,090,0,048$ e 0,022 respectivamente. Conclui-se que um menor campo de visão pode superestimar os valores do FVC de uma determinada área.

\section{MATERIAL E MÉTODOS}

A pesquisa foi desenvolvida na cidade de Cuiabá, capital do estado de Mato Grosso, situada na região Centro Oeste brasileira, localizada a $15^{\circ} 35^{\prime} 46^{\prime \prime}$ de latitude Sul e $56^{\circ} 05^{\prime} 48^{\prime \prime}$ de longitude Oeste, com altitude média de $165 \mathrm{~m}$. O perfil climático é o tropical semiúmido do tipo Aw, conforme classificação de Köppen-Geiger, caracterizado por precipitação elevada na primavera-verão e por seca acentuada no outono-inverno (MACHADO, et al. 2015).

A área selecionada para estudo foi o campus da Universidade Federal de Mato Grosso (UFMT), que ocupa uma extensão de 74 hectares, sendo caracterizado por um ambiente heterogêneo, com diferentes tipos de uso e ocupação do solo. Para a pesquisa de campo foram selecionados 09 pontos para o levantamento fotográfico (Figura 1). 


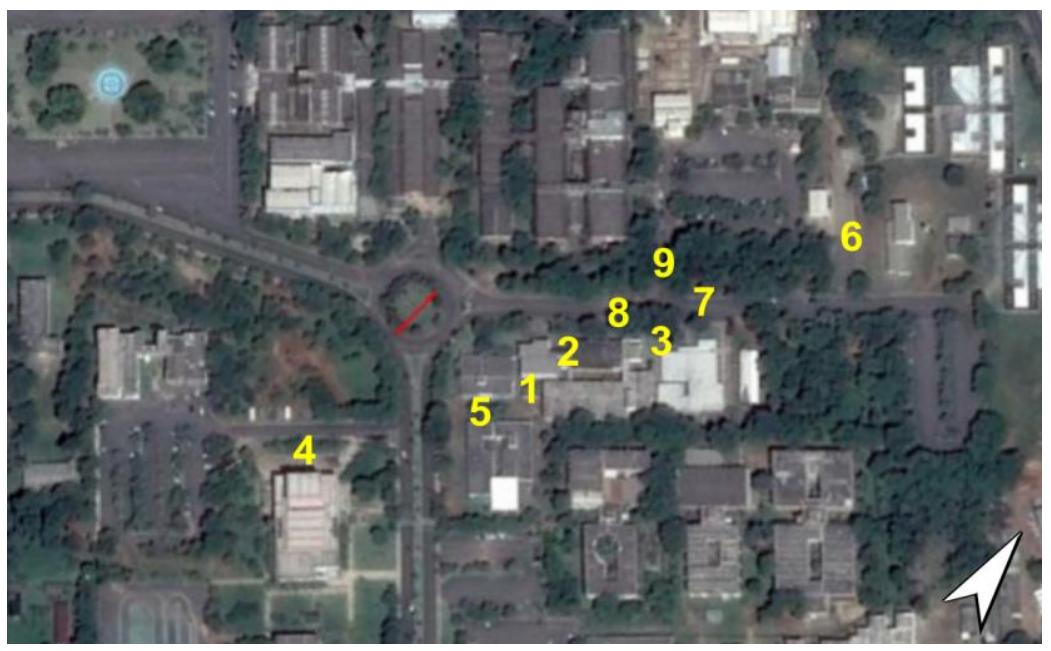

Figura 1 - Localização dos Pontos na área de estudo. Fonte: Imagem Google Earth adaptada pelos autores

\section{DELINEAMENTO DA PESQUISA}

O método empregado enquadra-se na pesquisa experimental, desenvolvida por meio dos seguintes procedimentos metodológicos: levantamento em campo das imagens hemisféricas de cada ponto, utilizando diferentes lentes olho-de-Peixe; estimativa do FVC e da radiação solar direta (RSD) incidente; análise quantitativa e qualitativa das diferenças dos valores do FVC, obtidos com as lentes alternativas em relação aos valores obtidos com o equipamento de referência.

Para avaliação comparativa dos equipamentos alternativos buscou-se a definição de equipamento referencial com base em pesquisas envolvendo o FVC (VILELA, et al 2009; SVENSSON, et al 2004; MINELLA, et al 2009b), sendo a câmera digital com sensor Full Frame e lente olho-de-peixe de $8 \mathrm{~mm}$, uma configuração amplamente utilizada. Para este estudo foi utilizado um modelo Nikon Coolpix-4300 e uma lente Nikon 8mm FC-E8, 0.21x, definido como equipamento de referência (ER).

$\mathrm{Na}$ definição das alternativas de baixo custo, foram observados os resultados apontados por Amorim et al. (2014), onde uma lente olho-de-peixe genérica de $180^{\circ}$ apresentou ângulo de abertura real de $160^{\circ}$. Assim, considerando uma possível limitação, para este estudo, foi definido a avaliação com duas lentes, uma lente olho-de-peixe de $180^{\circ}$ genérica acoplada a um smartphone, definida como equipamento alternativo EA-180 (Figura 2b) e uma lente olho-de-peixe de $235^{\circ}$ genérica acoplada ao mesmo smartphone, definida como equipamento alternativo EA-235 (Figura 2a). 


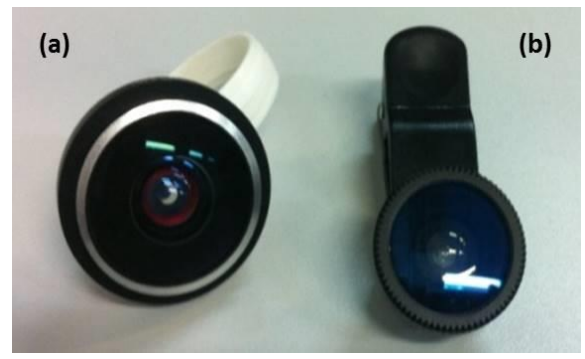

Figura 2 - Lentes olho-de-peixe alternativas para celulares. Fonte: Imagens produzidas pelos autores

Na primeira etapa foi realizado conjunto de imagens para selecionar os ajustes quanto ao enquadramento, conforme as possíveis configurações dos equipamentos testados (Figura $3 a, b, c)$. Na segunda etapa foi realizada avaliação dos ângulos de abertura das lentes, sendo executada em ambiente interno conforme os seguintes procedimentos: levantamento das dimensões da sala; do ponto central sobre o piso; da altura do equipamento ER (h); representação gráfica em escala da sala e equipamento; estimativa da altura das marcações no plano vertical para ângulos de $10^{\circ}$, conforme representação esquemática (Figura $3 \mathrm{~d}$ ); execução das imagens hemisféricas e avaliação visual dos ângulos de abertura dos equipamentos ER, E-180 e do E-250
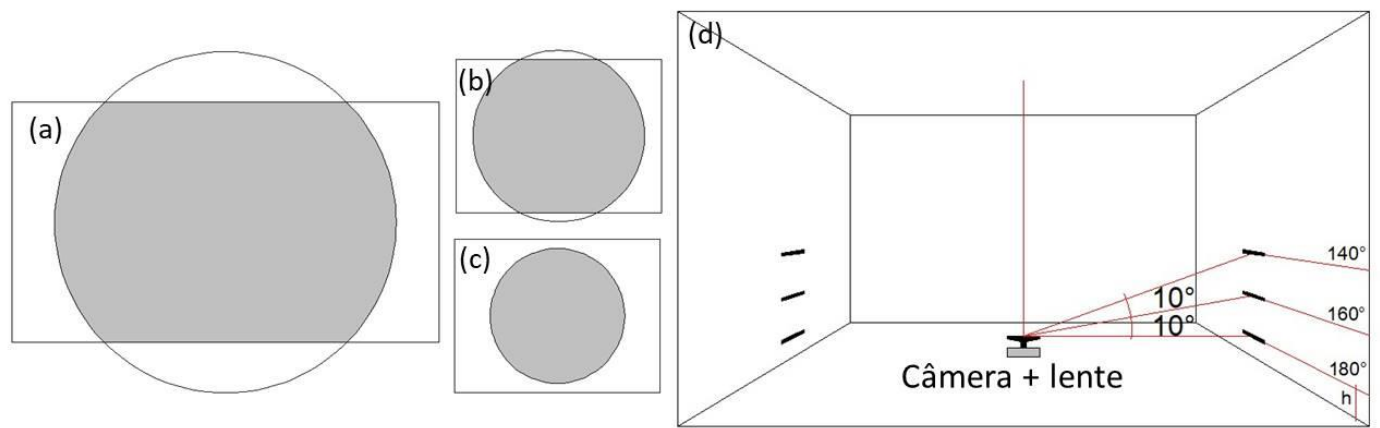

Figura 3 - Opções de enquadramentos dos equipamentos testados: alternativos (a) de $16 \times 9$, (b) de $4 \times 3$, (c) equipamento $E R$ de $4 \times 3$ e (d) representação esquemática da avaliação dos ângulos das lentes testadas.

Os pontos de observação foram definidos buscando-se áreas com diversidade quanto às configurações do entorno, sendo os pontos 1 , 2, e 3 em áreas limitadas por edificações; os pontos 4, 5 e 6 em áreas com edificações e elementos arbóreos e os pontos 7, 8 e 9 em áreas limitadas por copas arbóreas (Figura 4). 

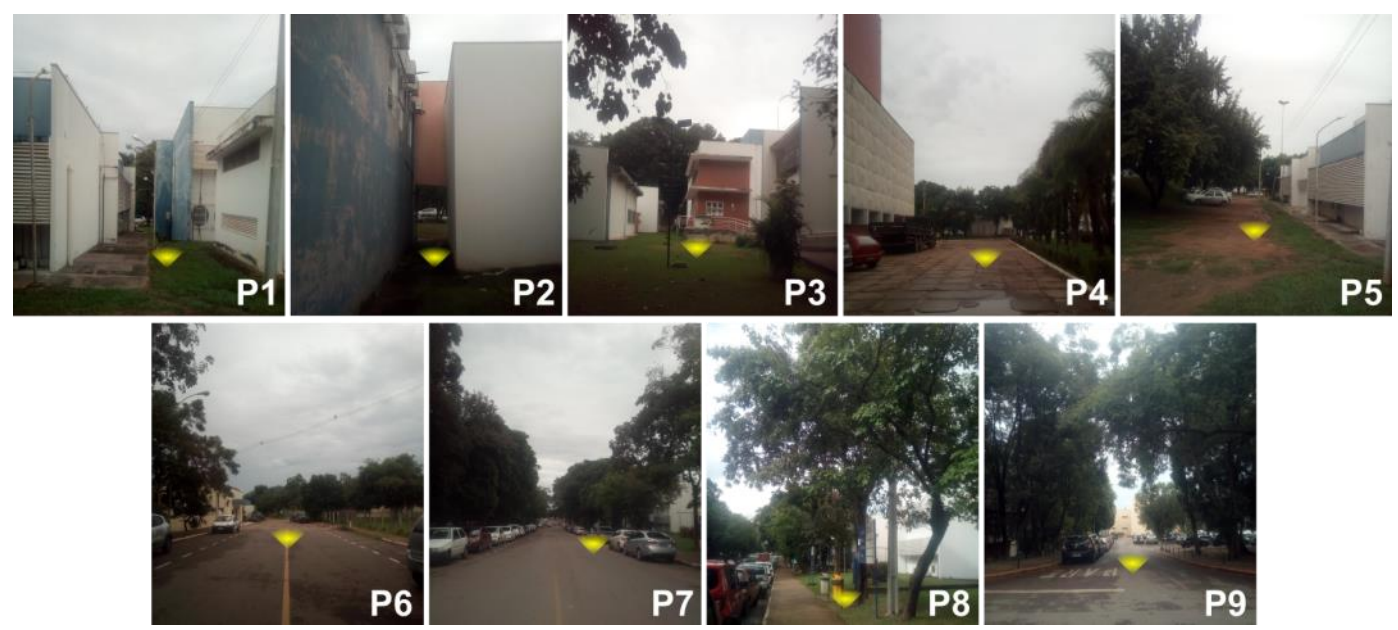

Figura 4 - Áreas e localização dos pontos selecionados para o levantamento das imagens hemisféricas. Fonte: Imagens produzidas pelos autores

Os pontos selecionados apresentaram relações geométricas semelhantes, conforme detalhado na Tabela 1.

Tabela 1 - Caracterização das áreas selecionadas para estimativa do FVC e a relação entre altura $(\mathrm{H})$ e largura (W) nos pontos avaliados

\begin{tabular}{clc}
\hline Pontos & \multicolumn{1}{c}{ Localização e descrição dos pontos } & Relação H x W \\
\hline 1 & Entre edificações de um pavimento & $\mathrm{H}>\mathrm{W}$ \\
2 & Passagem entre edificações de dois pavimentos & $\mathrm{H}>\mathrm{W}$ \\
3 & Área entre edificações de um e dois pavimentos & $\mathrm{H}>\mathrm{W}$ \\
4 & Centro da rua com um lado arborizado & $\mathrm{H}<\mathrm{W}$ \\
5 & Estacionamento com um lado arborizado & $\mathrm{H}<\mathrm{W}$ \\
6 & Centro de avenida edificação e arborização & $\mathrm{H}<\mathrm{W}$ \\
7 & Rua arborizada nos dois lados & $\mathrm{H}<\mathrm{W}$ \\
8 & Próximo à calçada sob copa arbórea & $\mathrm{H}<\mathrm{W}$ \\
9 & Rua arborizada de acesso a um estacionamento & $\mathrm{H}<\mathrm{W}$ \\
\hline
\end{tabular}

As imagens hemisféricas foram coletadas no mês de fevereiro de 2016, no início da manhã, devido às condições mais favoráveis, céu claro com ângulo de incidência solar baixo. Sendo os horários do inicio e do final do dia os mais indicados, devido ao contraste entre as cores ser mais elevado e evita-se a radiação direta na lente da câmera fotográfica (GRIMMOND et al., 2001).

A marcação do ponto de visão do céu, para captura das imagens, foi definida no centro da largura das áreas e no plano vertical optou-se pela avaliação em duas alturas, sendo um ponto de visão do céu sobre o solo $(0,0 \mathrm{~m})$ e um ponto acima do solo $(1,10 \mathrm{~m})$.

Destaca-se que o ponto de visão a $1,10 \mathrm{~m}$ do solo é empregado com frequência por diversos pesquisadores (MINELLA et al.2009b), em razão de a altura ser padrão para os sensores na aferição de variáveis microclimáticas, conforme a ISO 7726 (ISO, 1998). Porém, Grimmond et al. (2001) observaram 
que a influência do fator altura do ponto de visão não é discutido de forma mais ampla entre pesquisadores da área.

$\mathrm{Na}$ execução das imagens, com os E-180, E-250 e o ER, foram empregados um tripé para padronizar a altura de 1,10m e uma superfície plana, onde foi utilizada uma placa de isopor (EPS) para apoio dos equipamentos, nível e bússola. Assim, a cada ponto foram capturas seis imagens, totalizando 54 imagens hemisféricas da área de estudo. As imagens registradas foram tratadas no programa Inkscape, onde foram realizados ajustes de contraste e recorte para obtenção de um frame circular das imagens hemisféricas. As imagens finalizadas foram inseridas no programa RayMan (MATZARAKIS, et al. 2010), sendo obtidas as máscaras de obstrução sobrepostas à carta solar da área de estudo e os valores estimados para o FVC.

A avaliação qualitativa teve por objetivo a estimativa do efeito da variação do FVC em relação a uma variável afetada pela parcela de céu visível de uma determinada área. Por meio do programa RayMan foram estimados os valores da radiação solar direta (RSD) e as horas de incidência para o período anual observado.

A variação entre os valores do FVC e o fator altura, ângulo das lentes e a incidência da radiação solar foram avaliadas por meio de coeficientes de erro. 0 efeito do fator altura do ponto de visão e ângulos das lentes alternativas sobre os valores do FVC foram avaliados por meio da análise de covariância (ANCOVA) univariada, com o objetivo de avaliar, além dos efeitos dos fatores fixos, a necessidade de compensação a partir das covariáveis, assim como a comparação destas regressões para diferentes grupos. O modelo testado teve como variável dependente os valores do FVC do ER, como fatores fixos as alturas do ponto de visão e os ângulos das lentes alternativas, e como covariável os valores do FVC obtidos com os E-180 e E-250.

Os pressupostos de homocedasticidade e aderência dos resíduos à distribuição normal foram avaliados pelos testes de Levene e KolmogorovSmirnov, respectivamente, não detectando diferenças no nível de significância menores que $5 \%$.

\section{RESULTADOS E DISCUSSÃO}

Nos testes preliminares, foi possível identificar que nos equipamentos sem o sensor Full Frame a redução do enquadramento padrão de $16 \times 9 \mathrm{~cm}$ para $4 \times 3 \mathrm{~cm}$ proporcionou menor área de corte da imagem. Para o enquadramento $4 \times 3 \mathrm{~cm}$, o Frame circular da imagem apresentou área de corte mais reduzido nas duas extremidades do círculo, comparada a área de corte da imagem para o enquadramento mais retangular de $16 \times 9 \mathrm{~cm}$.

As limitações do fator de corte dos equipamentos alternativos, AE-180 e $\mathrm{AE}-235$, foram contornadas com o posicionamento das áreas de corte na direção das áreas com elementos de obstrução. Na edição das imagens as áreas do corte foram completadas, como pode ser observado nos diagramas hemisféricos (Figura 5 b, c, e, f) obtidos com os equipamentos alternativos, conforme apresentado em alguns dos pontos com elementos arbóreos. 


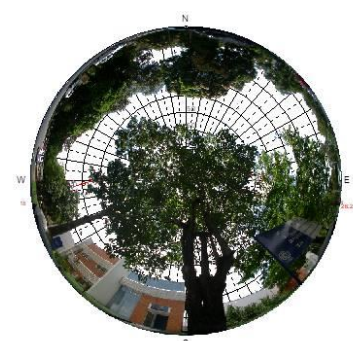

(a) P.8-ER $(1,10 \mathrm{~m})$

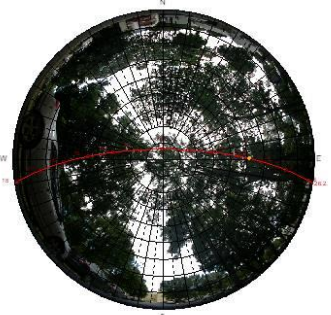

(d) P.9-ER $(0,0 \mathrm{~m})$

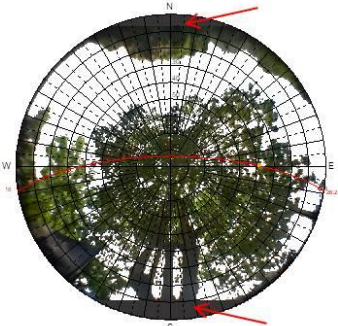

(b) P.8-EA-180(1,10m)

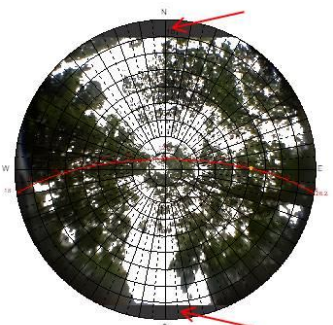

(e) P.9-EA-180(0,0m)

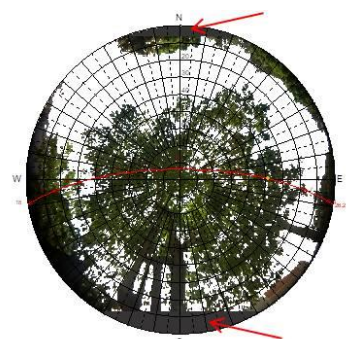

(c) P.8-EA-235(1,10m)

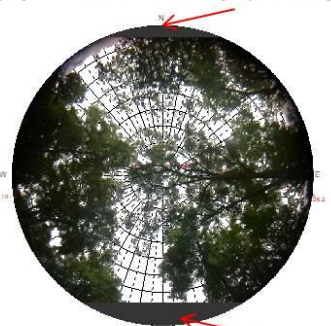

(f) P.9-EA-235(0,0m)

Figura 5 - Diagramas hemisféricos dos pontos com vegetação, obtidos com o equipamento de referência (ER) e os equipamentos alternativos (EA-180 e EA-235) em duas alturas, no solo $(0,0 \mathrm{~m})$ e acima $(1,10 \mathrm{~m})$

Quanto ao ângulo de abertura, para as lentes alternativas foi observado um ângulo real menor que o especificado. A lente alternativa de $180^{\circ}$ apresentou uma redução aproximada de $15^{\circ}$, ou seja, a abertura angular estimada foi de $165^{\circ}$. Para a lente alternativa de $235^{\circ}$, a redução observada foi de aproximadamente $75^{\circ}$, com abertura angular real de $160^{\circ}$. Para a lente de referência, as imagens observadas confirmaram a abertura real de aproximadamente $180^{\circ}$.

O efeito proporcionado pelos diferentes ângulos de abertura das lentes testadas pode ser visualizado nos diagramas hemisféricos de alguns dos pontos avaliados (Figura 5). Para o equipamento ER o registro das áreas de obstrução foi maior, comparado ao registrado pelos equipamentos alternativos, EA-180 e EA-235, com ângulos de abertura efetivos menores que $180^{\circ}$, conforme diagramas hemisféricos (Figura $6 a, d, g, j$ ). 


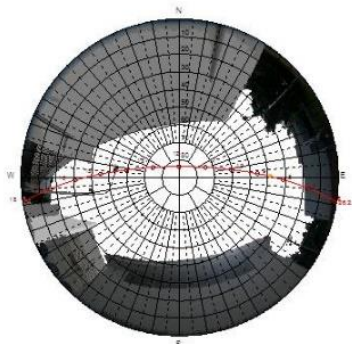

(a) P.1-ER(1,10m)

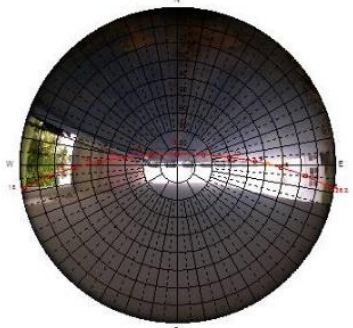

(d) P.2-ER(0,0m)

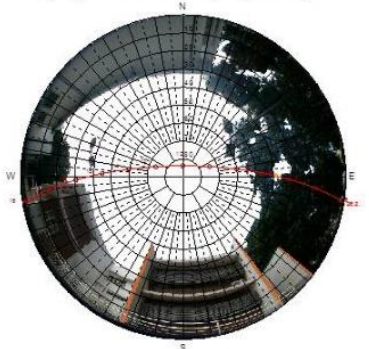

(g) P.3-ER(1,10m)

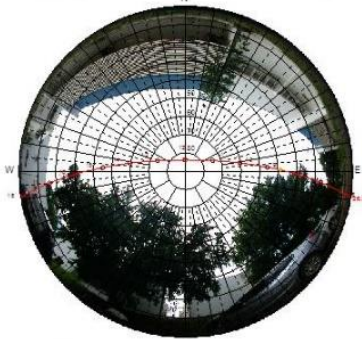

(j) P.5-ER $(0,0 m)$

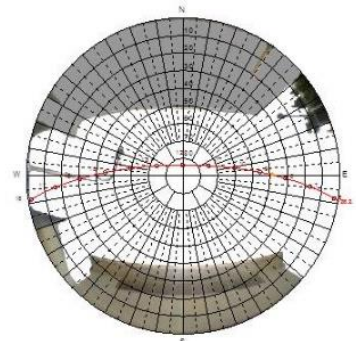

(b) P.1-EA-180 $(1,10 \mathrm{~m})$

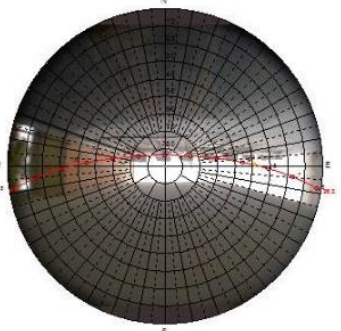

(e) P.2-EA-180 $(0,0 \mathrm{~m})$

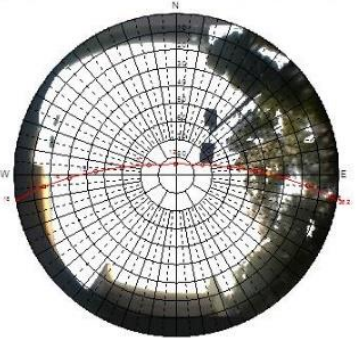

(h) P.3-EA-180 $(1,10 \mathrm{~m})$

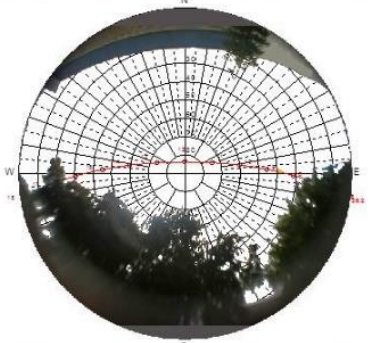

(I) P.5-EA-180 $(0,0 \mathrm{~m})$

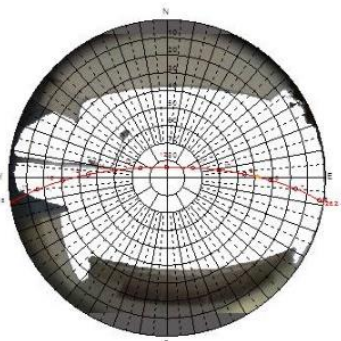

(c) P.1-EA-235(1,10m)

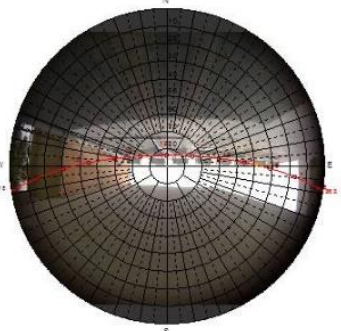

(f) P.2-EA-235(0,0m)

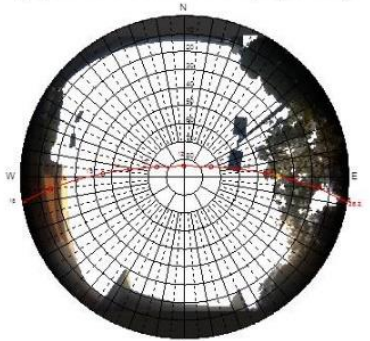

(i) P.3-EA-235(1,10m)

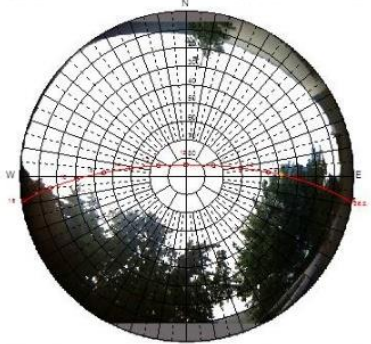

(m) P.5-EA-235 $(0,0 \mathrm{~m})$

Figura 6 - Diagramas hemisféricos obtidos com o equipamento de referência com lente de $180^{\circ}$ (ER), os equipamentos alternativos (EA-180 e EA-235) e com o ponto de visão em duas alturas, no solo $(0,0 \mathrm{~m})$ e acima do solo com $(1,10 \mathrm{~m})$

Na estimativa dos valores do FVC (Figura 7 a, b), para as variações de equipamento e altura, o ER apresentou os menores valores, comparados aos valores do FVC dos equipamentos alternativos EA-180 e EA-235. O ponto 2 apresentou a menor parcela de céu visível com FVC médio de 0,042(0,0m) e $0,047(1,10 \mathrm{~m})$, seguido do ponto 9 com médias de $0,122(0,00)$ e $0,127(1,10)$, sendo área com elevada cobertura arbórea. Os valores máximos do FVC foram registrados no ponto 6 com médias de $0,694(0,0 \mathrm{~m})$ e $0,695(1,10 \mathrm{~m})$, seguido do ponto 4 com médias de $0,507(0,00)$ e $0,543(1,10)$ conforme Figura 6 (a) (b). 
De modo geral, para equipamentos e alturas, nos pontos 1, 2 e $3 \mathrm{com}$ relação $\mathrm{H}>\mathrm{W}$ a média do FVC foi menor, em comparação a média dos pontos 4, 5, 6 em áreas com relação $\mathrm{H}<\mathrm{W}$, onde os pontos 7, 8 e 9 apresentaram redução expressiva do FVC devido a maior cobertura arbórea nos pontos.
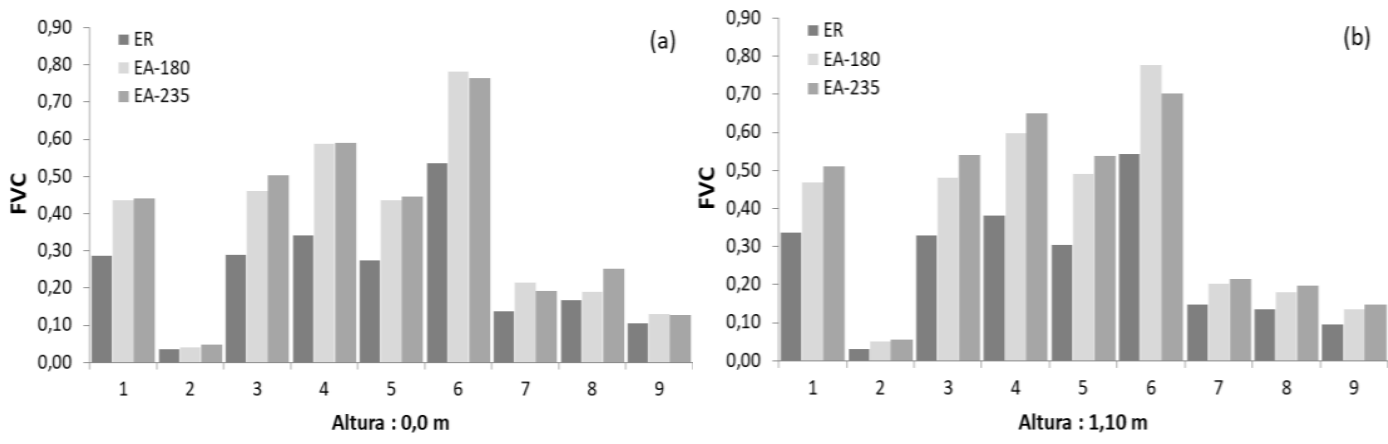

Figura 7 - Estimativa do FVC com os equipamentos de referência (ER) e os alternativos (EA-180 e EA-235) obtidos em duas alturas (a) no nível do solo e (b) acima do solo

Na avaliação comparativa dos valores do FVC obtidos com o equipamento de referência ER e os equipamentos alternativos, as diferenças foram menores para o EA-180, em relação aos percentuais do erro médio relativo (EMR) para as duas alturas. Porém, observa-se que as diferenças entre os equipamentos alternativos foram ampliadas com o deslocamento do ponto de visão para $1,10 \mathrm{~m}$ de altura, conforme Tabela 2.

Tabela 2 - Estimativa do erro absoluto (EA) mínimo, máximo e o erro médio relativo (EMR) dos valores do FVC, na comparação entre o equipamento de referência e os alternativos (EA-180 e EA-235) em cada altura do ponto de visão, e a comparação entre as alturas para o equipamento de referência e os equipamentos alternativos.

\begin{tabular}{ccccc}
\hline Altura $(\mathrm{m})$ & Equipamentos & EA min. & EA max. & EMR\% \\
\hline 0,00 & EA-180 & 0,007 & 0,247 & 44,4 \\
0,00 & EA-235 & 0,012 & 0,248 & 49,3 \\
1,10 & EA-180 & 0,020 & 0,232 & 46,5 \\
1,10 & EA-235 & 0,023 & 0,268 & 55,8 \\
\hline Comparação entre alturas & & & \\
\hline 0,00 e 1,10 & ER & $-0,009$ & 0,048 & 2,7 \\
0,00 e 1,10 & EA-180 & $-0,008$ & 0,055 & 4,6 \\
0,00 e 1,10 & EA-235 & $-0,055$ & 0,091 & 7,5 \\
\hline
\end{tabular}


Quanto aos efeitos da variação na altura do ponto de visão sobre os valores do FVC, apresentados na Tabela 2, as diferenças entre o ER e o EA-180 foram menores, em comparação às diferenças em relação ao EA-235, com maior percentual do EMR entre os equipamentos avaliados.

Os resultados da avaliação dos equipamentos, em relação aos valores do FVC de referência, demostram maior diferença entre os valores obtidos a 1,10m de altura. A elevação do ponto de visão proporcionou redução no registro das áreas de obstrução, gerando aumento da área de céu visível. O mesmo efeito ocorreu com a redução do ângulo de visão, de $180^{\circ}$ para $165^{\circ}$ e $160^{\circ}$, o que resultou em maior diferença do EA-235 em relação ao EA-180 e destes em comparação aos valores do FVC obtidos com o ER.

Os efeitos do ângulo de visão sobre os valores do FVC foram compatíveis com os valores encontrados por Minella et al. (2009), onde as diferenças entre câmeras e lentes profissionais, de $180^{\circ}$ e $160^{\circ}$, apresentaram erros absolutos de 0,10 a 0,29 com erro médio relativo percentual de $59,0 \%$ entre os valores do FVC de 14 pontos em áreas urbanas.

Com relação à altura do ponto de visão do céu, a variação entre o nível zero e a 1,10m acima do solo, para os valores do FVC as diferenças não atingiram $8 \%$. Observa-se que resultados encontrados para alturas maiores, apontaram diferenças significativas, como as encontradas por Grimmond et al. (2001) entre o nível zero e a 1,40m de altura com erro médio de $9 \%$, assim como as observadas por Svensson (2004), que identificou diferenças média acima de $10 \%$ entre os valores do FVC tomados no nível zero $(0,0 \mathrm{~m})$ e a $2,0 \mathrm{~m}$ de altura, em levantamento com câmera e lente profissionais.

Pontualmente o efeito da variação dos equipamentos e das alturas sobre - FVC foi diferenciado entre as áreas avaliadas, sendo que, nas áreas com relação de $\mathrm{H}>\mathrm{W}$, pontos $1,2,3$, apresentaram diferenças do FVC com EMR de $47,6 \%$. Para as áreas com relação de $\mathrm{H}<\mathrm{W}$, pontos 4,5 e 6, o EMR estimado foi de $57,6 \%$, entretanto, para os pontos 7, 8, 9 mesmo estando localizados em áreas com relação de $\mathrm{H}<\mathrm{W}$ a área de obstrução da abóboda celeste foi ampliada, devido às copas das árvores, gerando redução nas diferenças do FVC com percentual do EMR de 37,9\%.

Os resultados observados corroboram com as observações de Grimmond et al. (2001), sobre o impacto das variações do ângulo das lentes serem mais acentuados em áreas com relação de $\mathrm{H}<\mathrm{W}$, ou seja, altura dos planos de obstrução menor que a largura total da área avaliada, não considerando a obstrução da abóboda celeste por copa de árvores.

\section{ANÁLISE ESTATÍSTICA}

$\mathrm{Na}$ análise univariada o coeficiente de determinação foi de 0,99, porém foram detectadas diferenças significativas $(p<5 \%)$ entre a covariável (valores do FVC dos E-180 e E-235) e para interação entre a covariável e os fatores fixos (altura e o ângulo das lentes alternativas). No entanto, não foram detectadas diferenças significativas entre as tendências centrais dos valores do FVC estimados pelos dois tipos de lentes alternativas, assim como, entre os valores estimados para as diferentes alturas do ponto de visão, sendo a opção entre os equipamentos de cunho qualitativo. 
Para tanto, buscou-se ponderações a partir da interpretação das imagens hemisféricas e dos respectivos valores do FVC, uma vez que não foi identificado paralelismo entre as tendências lineares para os tipos de lentes alternativas (interação entre fator fixo e covariável). Desse modo buscou-se avaliação por meio de regressão linear simples com bootstrap, utilizando 1000 reamostragens com intervalo de confiança de $95 \%$, para os dois equipamentos alternativos, EA180 e EA-235, apresentado na Tabela 3.

Tabela 3 - Correlação entre os valores do FVC do equipamento de referência (ER) e os alternativos (EA-180 e EA-235)

\begin{tabular}{lcccc}
\hline Modelo 1 & ajustado & $\begin{array}{c}\text { Coeficiente } \\
\text { linear }\end{array}$ & $\begin{array}{c}\text { Coeficiente } \\
\text { angular }\end{array}$ & $\begin{array}{c}\text { Nível de } \\
\text { Significância }\end{array}$ \\
\hline EA-180 & 0,978 & 0,008 & 0,648 & 0,000 \\
EA-235 & 0,951 & 0,007 & 0,636 & 0,000 \\
Variável Dependente: ER & & \\
\hline
\end{tabular}

Os valores do FVC obtidos com as propostas alternativas apresentaram correlação com os valores de referência ER, sendo maior a correlação com o equipamento E-180 comparada à correlação com o equipamento E-235, porém, com precisão significativa dos coeficientes de determinação para as duas alternativas. Valores elevados para o coeficiente de determinação, também foram observados por Minella et al. (2009), que identificaram uma correlação linear R2 de 0,98 entre os valores de FVC obtidos com lentes olho-de-peixe de $180^{\circ}$ e de $160^{\circ}$ em equipamentos profissionais.

A avaliação qualitativa dos equipamentos alternativos pressupôs a verificação de variável afetada pelo FVC, como a incidência da radiação solar (Figura 8 a, b) definida pelo FVC em razão deste expressar a parcela da abóboda celeste disponível para entrada de radiação solar em determinado ponto da área avaliada, deste modo, foi avaliada a estimativa das horas em que a área pode receber a incidência da radiação solar direta (RSD), sob condições atmosféricas favoráveis. 

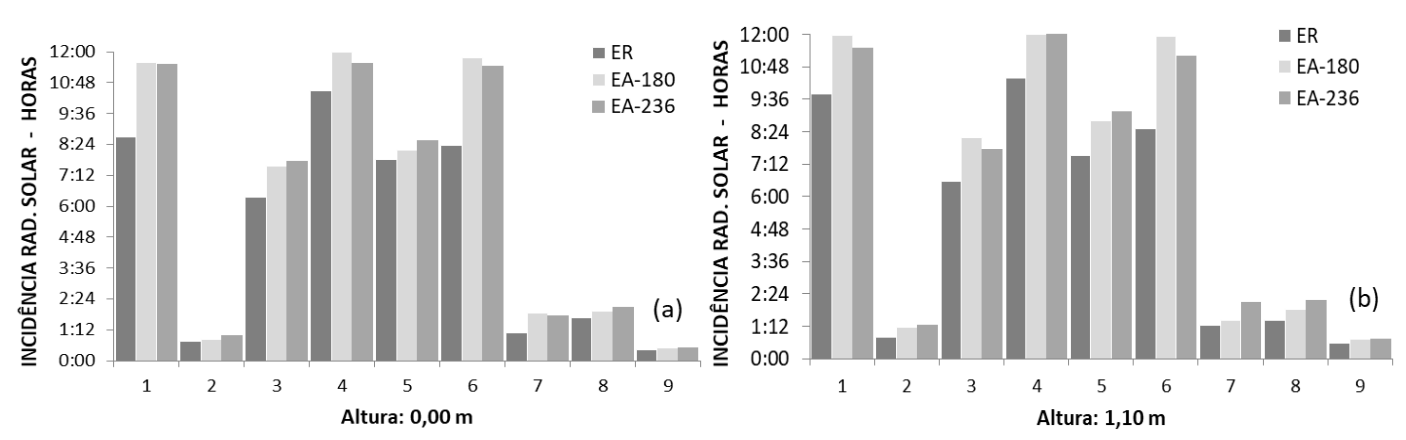

Figura 8 - Estimativa das horas de incidência da radiação solar direta para os equipamentos de referência (EA) e os alternativos (EA-180 e EA-235) obtidos em duas alturas (a) no nível do solo e (b) acima do solo

O potencial da incidência da RSD apresentou comportamento semelhante em relação aos períodos (horas) de incidência máxima e mínima, ou seja, os pontos com valores máximos e mínimos não foram alterados pela variação dos equipamentos e pela variação das alturas do ponto de visão. Os maiores valores, estimados em horas de incidência, foram para o ponto 4 (FVC de 0,342 a 0,650), seguido do ponto 1 (FVC de 0,289 a 0,511) e do ponto 6 (FVC de 0,536 a 0,783), respectivamente. O período mínimo de incidência foi estimado para o ponto 9 (FVC de 0,097 a 0,148), seguido do ponto 2 (FVC de 0,033 a $0,056)$.

Observa-se, que os pontos com incidência máxima (p.4) e mínima (p.9) não corresponderam aos valores do FVC máximos (p.6) e mínimos (p.2). No ponto 4 a posição da área obstruída não bloqueou a incidência da RSD, as diferenças entre as horas de incidência nos pontos 4 e 6 variaram de 0:05h a 2:08h. Entre os pontos 9 e 2 as diferenças foram reduzidas com variação de $0: 14 \mathrm{~h}$ a $0: 30 \mathrm{~h}$ e podem ser atribuídas a imprecisão em quantificar penetração da radiação solar direta em copa de àrvores menos densas, como as do ponto 9.

Na avaliação do total de horas de incidência da RSD estimada pelo FVC, em comparação ao ER e os equipamentos alternativos, apresentados na Tabela 4, as diferenças médias apresentaram menor variação e não ultrapassam 1:15h. Os erros absolutos mínimos foram estimados para o ponto 9 e os erros máximos para o ponto $6 \mathrm{com}$ maior valor do FVC. O equipamento EA-180 apresentou menor acréscimo percentual, em relação às horas de incidência do equipamento ER, comparado aos percentuais estimados para o equipamento EA-235. Para os equipamentos alternativos as diferenças entre as horas de incidência da RSD foram maiores para o ponto de visão a $1,10 \mathrm{~m}$ de altura, sendo mais acentuadas as diferenças para o EA-235, com maior percentual para o EMR. 
Tabela 4 - Estimativas dos erros absoluto (EA) mínimos, máximos, médio (EMA) e o erro relativo médio (EMR\%) das horas de incidência da radiação solar, na comparação entre os equipamentos de referência e os alternativos, e a comparação entre as alturas.

\begin{tabular}{cccccc}
\hline Alturas (m) & Equip. & EA.min. & EA.max. & EMA & EMR\% \\
\hline 0,00 & EA-180 & $0: 05$ & $3: 24$ & $1: 10$ & 25,4 \\
0,00 & EA-235 & $0: 05$ & $3: 06$ & $1: 10$ & 28,2 \\
1,10 & EA-180 & $0: 09$ & $3: 25$ & $1: 14$ & 26,2 \\
1,10 & EA-235 & $0: 11$ & $2: 43$ & $1: 13$ & 35,0 \\
\hline Comparação entre alturas & & & \\
\hline 0,00 e 1,10 & ER & $-0: 07$ & $1: 06$ & $0: 48$ & 10,7 \\
0,00 e 1,10 & EA-180 & $-0: 06$ & $0: 38$ & $0: 21$ & 14,7 \\
0,00 e 1,10 & EA-235 & $0: 07$ & $0: 31$ & $0: 18$ & 12,1 \\
\hline
\end{tabular}

$\mathrm{Na}$ avaliação comparativa entre as alturas do ponto de visão sobre os valores horários da RSD, apresentados na Tabela 2, as diferenças mínimas e máximas foram reduzidas, destacando as diferenças para o $E R$, com o EMA maior que os valores médios estimados para os equipamentos alternativos. Porém, para o ER o erro percentual médio foi menor, entre as diferenças dos valores horários proporcionado pela variação da altura do ponto de visão, comparado aos percentuais do EMR para os equipamentos alternativos.

Em alguns pontos o efeito mais acentuado do ER em relação à variação da altura, se deve ao maior ângulo de abertura da lente de $180^{\circ}$, que possibilitou o registro de maior área de obstrução em relação às lentes alternativas com menor ângulo de abertura, como pode ser observado nos menores valores do FVC para o ER. Desse modo, o deslocamento do ponto de visão afetou em maior proporção a redução da área de obstrução, como também pode ser observado no EMA entre os equipamentos alternativos, onde as diferenças para o EA-180 com ângulo de $165^{\circ}$ foram maiores que as diferenças para o EA-235 com ângulo de $160^{\circ}$.

\section{CONCLUSÕES}

Este estudo analisou o desempenho de equipamentos alternativos, lentes olho-de-peixe genéricas acopladas a uma câmera de smartphone, em relação aos efeitos sobre os valores do FVC em diferentes espaços urbano, tendo-se como referência, as imagens hemisféricas obtidas por uma câmera Nikon Coolpix-4300 com lente olho-de-peixe, modelo FC-E8.

As lentes olho-de-peixe genéricas testadas apresentaram ângulo de abertura menor que os especificados, com redução de $15^{\circ}$ para a lente de $180^{\circ}$ e de $75^{\circ}$ para a lente de $235^{\circ}$, em valores aproximados, ou seja, ângulos de visão menores que a do equipamento de referência com lente olho-de-peixe de 
$180^{\circ}$. O menor campo de visão registrado com as lentes alternativas proporcionou diferenças no registro das imagens hemisféricas e sobre os valores do FVC, que de modo geral apresentaram acréscimo médio de 44,4\% a 55,8\%, em relação aos valores do FVC de referência obtidos no nível do solo e a 1,10m acima do solo. Entretanto, a avaliação quantitativa não reportou diferenças estatisticamente significativas entre os efeitos proporcionados pelos diferentes ângulos das lentes alternativas.

A avaliação comparativa entre as alturas do ponto de visão não reportou efeitos significativos sobre os valores do FVC. Para os equipamentos alternativos os efeitos sobre o FVC obtido a $1,10 \mathrm{~m}$ de altura foram maiores, com diferenças de $4,6 \%$ a $7,5 \%$, em relação à diferença de $2,7 \%$ estimada para as diferenças médias do equipamento de referência.

Entretanto, os resultados da avaliação qualitativa dos equipamentos alternativos apresentaram efeitos significativos sobre os valores do FVC, com aumento médio de $25,4 \%$ a $35,0 \%$ sobre as horas diárias de incidência da radiação solar direta nos pontos avaliados. Em relação à variação na altura do ponto de visão, o efeito sobre as horas de incidência proporcionou aumento médio de $12,1 \%$ a $14,7 \%$ para os equipamentos alternativos e de $10,7 \%$ para o equipamento de referência. Observa-se que, em termos absolutos as diferenças máximas variaram de $0: 38 \mathrm{~h}$ a $1: 06 \mathrm{~h}$, o que reforça as observações quanto ao reduzido efeito sobre os valores do FVC devido a elevação do ponto de visão a $1,10 \mathrm{~m}$ acima do solo.

De acordo com os resultados alcançados conclui-se que, as lentes alternativas testadas não apresentaram desempenho satisfatório na obtenção do FVC por método fotográfico, sendo que, devido à condição genérica do produto não há especificação técnica que assegure a proposição de um fator para correção das diferenças em relação ao ângulo de visão.

\section{REFERÊNCIAS}

ALCOFORADO, M. J.; ANDRADE, H.; LOPES, A.; VASCONCELOS, J. Application of climatic guidelines to urban planning. The example of Lisbon-Portugal. Landscape and Urban Planning, n.90, p.56-65, 2009.

AMORIM, R. P. L; PEDROSA, M. L.; CARVALHO, H. J. M. Máscara de obstrução: uso de novas tecnologias que facilitam a verificação da obstrução do céu visível. XV Encontro Nacional de Tecnologia do Ambiente Construído, Maceió AL, p. 370378, 2014.

CHAPMAN, L. Towards a tree-view factor. Why do urban climatologists dislike trees. IAUC Newsletter, v. 25, p. 4-6, 2007.

GRIMMOND, C.S.B.; POTTER, S.K.; ZUTTER H.N.; SOUCH, C. Rapid methods to estimate sky-view factors applied to urban areas. Int J Climatol. International Journal of Climatology, n. 21, p. 903-913, 2001.

INTERNATIONAL ORGANIZATION FOR STANDARDIZATION. ISSO 7726: Thermal environments-instruments and methods for measuring physical quantities. Switzerland, 1998.

MACHADO, N. G.; BIUDES, M. S.; QUERINO, C. A. S.; DANELICHEN, V. H. de M.; VELASQUE, M. C. S. Seasonal and interannual pattern of meteorological 
variables in Cuiabá, Mato Grosso State, Brazil. Revista Brasileira de Geofísica, v. 33 n.3, p.1-10, 2015.

MATZARAKIS, A.; MATUSCHEK, O. Sky view factor as a parameter in applied climatology: rapid estimation by the SkyHelios model. Meteorologische Zeitschrift, v. 20, n.1, p. 039-045, 2011.

MATZARAKIS A.; RUTZ F.; MAYER H. Modelling radiation fluxes in simple and complex environments: basics of the RayMan model. International Journal of Climatology, p.131-139, 2010.

MINELLA, F. O.; TAMURA, C. A.; KRÜGER, E. L. Apresentação de método fotográfico para obtenção do fator de visão do céu. In: XI Encontro Nacional e IX-Encontro Latino-Americano de Conforto no Ambiente Construído, 2009, Natal RN, Anais... Natal, ANTAC, 2009a.

MINELLA, F. O.; ROSSI, F. A.; KRÜGER E. L. Influência do fator de visão do céu no conforto térmico em duas situações urbanas distintas. $X$ Encontro Nacional e VI Encontro Latino Americano de Conforto no Ambiente Construído, Natal, RN, 2009b.

NAKATA-OSAKI, C. M.; SOUZA, L. C. L. de; RODRIGUES, D. S. Impacto da geometria do cânion urbano na intensidade de ilha de calor noturna: análise através de um modelo simplificado adaptado a um SIG. Ambiente Construído, Porto Alegre RS, v.16, n.3, p.73-87, 2016.

OKE, T. R. Canyon geometry and the nocturnal urban heat island: comparison of scale model and field observations. Journal of Climatology, n.1, p. 237-254, 1981.

RIBEIRO, C. A. de M.; CARVALHO, H. J. M. de; SANTOS, M. A. dos. Metodologia para análise do sombreamento em centros urbanos. III Simpósio Brasileiro de Ciências Geodésicas e Tecnologias da Geoinformação, Recife, PE, p. 001-009, 2010.

SOUZA, L. C. L. de; RODRIGUES, D. S.; MENDES, José Fernando G. Expandindo um SIG-3D para aplicação em conforto ambiental em nível urbano. ENCAC COTEDI, Curitiba, PR, p. 669-676, 2003.

SOUZA, L. C. L. de; TENTE, C. M.; GIUNTA, M. B.; NAKATA, C. M. Fator de visão do céu e intensidade de ilhas de calor na escala do pedestre. Ambiente Construído, Porto Alegre RS, v.10, n.4, p.155-167, 2010.

SVENSSON, Marie K. Sky view factor analysis - implications for urban air temperature differences. Meteorol. Appl. n.11, p. 201-211, 2004.

VILELA, J. A; ASSIS, E. S; PAIVA, J. E. M. de. Variáveis do clima urbano: análise da situação atual e prognósticos para a região do bairro Belvedere III, Belo Horizonte, MG. X Encontro Nacional e VI Encontro Latino Americano de Conforto no Ambiente Construído, Natal, RN, 2009 